\title{
Transfer function measured by electromagnetic sounding with an accurately controlled signal
}

\author{
Yukiko Yokoyama, Mineo Kumazawa, and Takahiro Nakajima
}

Tono Geoscience Center, Japan Nuclear Cycle Development Institute, Japan

(Received February 15, 2001; Revised December 3, 2001; Accepted February 6, 2002)

\begin{abstract}
We present the fundamentals of an electromagnetic sounding with an accurately controlled source signal. In this method, the tensor transfer function between transmitted and received vector electromagnetic fields is measured. We formulated the transfer function by introducing a ray model that describes the propagation of the general electromagnetic field in the whole frequency region. The transfer function includes information of the underground structure: electromagnetic parameters, propagation distance, and boundary planes. The information of the electromagnetic parameters and the propagation distance is commonly described in all the tensor elements. It is in a form of complex functions of frequency. The information concerning the boundary planes is described as real coefficients of the complex functions. The coefficients are different for each of the tensor elements. When we use an accurately controlled signal, we can estimate the information as a linear inversion problem. A profile of the transfer function varies with the frequency region: it decays rapidly in the low-frequency region and oscillates in the high-frequency region. Such a profile can be explained by the approximate form of the transfer function: a sum of complex exponential functions in a narrow frequency band. The approximate form also provides a good model for data decomposition.
\end{abstract}

\section{Introduction}

Electromagnetic-sounding methods with controlled source signals are useful for exploration of the underground (Strack, 1992; Zhdanov and Keller, 1994). Controlled source audio-frequency magnetotellurics (CSAMT) is a well-known method and measures the impedance tensor (Kaufman and Keller, 1983). Transient electromagnetics (TEM) and ground penetrating radar (GPR) are also popular methods. Signals used are impulse, sinusoidal waves, and pseudo-random sequences, and the electromagnetic field is commonly measured in the time domain (Duncan et al., 1980; Strack, 1992).

The electromagnetic accurately controlled routinely operated signal system (EM ACROSS) is based on the same principle as TEM and GPR. Ogawa and Kumazawa (1996), Kumazawa (1998a, b), and Nakajima et al. (1998) first proposed the concept of EM ACROSS and developed the method with an accurately controlled continuous signal consisting of a finite number of continuous sinusoids. The signal employed is different from those previously used, especially concerning the accuracy of the transmitted signal and synchronization of transmitted and received signals. A wave form of the signal is not tightly constrained and can be designed to obtain an ideal signal level in the frequency domain (Yokoyama et al., 2000b).

The first experimental system of the EM ACROSS was developed by Nakajima et al. (2000). They succeeded in transmitting an accurately controlled rectangular wave

Copy right (C) The Society of Geomagnetism and Earth, Planetary and Space Sciences (SGEPSS); The Seismological Society of Japan; The Volcanological Society of Japan; The Geodetic Society of Japan; The Japanese Society for Planetary Sciences. signal and achieved synchronization between receiver and transmitter with a timing accuracy from $10^{-8}$ to $10^{-6} \mathrm{~s}$. Using the signal, the signal to noise ratio (SNR) of the received signal increased in proportion to the square-root of the stacking duration, as theoretically expected, and a high SNR was obtained. This advantage is not provided when a non-accurately controlled signal was used because the averaged signal level would not increase by stacking, as expected from theory. Accordingly, a high SNR is one advantage of using the accurately controlled signal.

Another advantage of the accurately controlled signal is that it allows measurement of the transfer function between transmitted and received signals. Our purpose in this paper is to show this advantage. To achieve this, firstly we clarify the reason why the transfer function can be measured. Secondly, we describe the relation between an electromagnetic structure and the transfer function in order to clarify the physical meaning of the transfer function. Then we show numerical examples of the transfer function and explain their frequency profiles in a narrow frequency band. Finally, the advantageous points of the present approach are summarized. This study is also a fundamental study of the theory of electromagnetic sounding with an accurately controlled signal.

\section{The Transfer Function}

In this section, we show the reason why the transfer function can be measured in the case of an accurately controlled signal. We consider the case where a controlled electromagnetic signal $x^{0}(n)$, sampled at discrete time $n$, is transmitted into the underground. The signal propagates in the ground 
and is observed as $y^{o b s}(n)$ at the receiving point. The received signal has a decayed amplitude and delayed phase compared to the transmitted signal. The amplitude decay and the phase delay together are described as the transfer function $h(n)$, which depends on both the frequency of the signal and the electromagnetic structure of the underground. The transfer function is set to be time-invariant by assuming that the electromagnetic structure does not change during the observation.

When we use an ordinary controlled signal, the actually transmitted signal $x(n)$ is a sum of the known signal $x^{0}(n)$ and a transmitting noise $\varepsilon_{x}(n)$ :

$$
x(n)=x^{0}(n)+\varepsilon_{x}(n) .
$$

The transmitting noise includes and a control error caused by a clock error, environmental noise, dielectric polarization of contacting place, state change of contacting place, and so on. The received signal $y^{o b s}(n)$ includes observation noise $\varepsilon_{y}(n)$ in addition to the propagated signal $y(n)$ :

$$
y^{o b s}(n)=y(n)+\varepsilon_{y}(n),
$$

where

$$
y(n)=\sum_{m=-\infty}^{\infty} x(m) h(n-m) .
$$

In this case, the system of the transmitted and received signals is described as in Fig. 1(a). Because the observable quantity $y^{o b s}(n)$ includes unknown parameters $\varepsilon_{x}(n), h(n)$, and $\varepsilon_{y}(n)$, we should estimate the transfer function $h(n)$ using a proper model. This estimation is a non-linear problem because the unknown parameters $\varepsilon_{x}(n)$ and $h(n)$ are convoluted in the observation $y^{o b s}(n)$ (Oppenheim and Schafer, 1975). On the other hand, when we use the accurately controlled signal, the estimation problem becomes linear. In this paper, we define the accurately controlled signal by the following two conditions:

$$
\left|\frac{E_{x}(\omega)}{X^{0}(\omega)}\right| \ll 1
$$

and

$$
\left|\frac{E_{x}(\omega)}{X^{0}(\omega)}\right| \ll\left|\frac{E_{y}(\omega)}{Y^{o b s}(\omega)}\right|,
$$

where $X^{0}(\omega), E_{x}(\omega), Y^{o b s}(\omega)$, and $E_{y}(\omega)$, are the Fourier transforms of $x^{0}(n), \varepsilon_{x}(n), y^{o b s}(n)$, and $\varepsilon_{y}(n)$, respectively. These conditions indicate a small transmitting noise $\varepsilon_{x}(n)$. This noise can be suppressed by using more stable hardware and an accurate clock. Monitoring of the transmitted signal also decreases the control error because the monitored data can be used to convert some fraction of the unknown error into known signal $x^{0}(n)$.

With the accurately controlled signal, the condition

$$
\left|\frac{E_{y}(\omega)}{Y^{o b s}(\omega)}\right| \ll 1
$$

can be efficiently achieved by stacking (Nakajima et al., 2000). EM ACROSS is designed to satisfy this condition.
The conditions of Eqs. (4) and (6) are necessary to make the problem linear. With the conditions, the Fourier transform $H(\omega)$ of the transfer function in Eq. (3) derived from Eqs. (1)-(2),

$$
H(\omega)=\frac{Y^{o b s}(\omega)-E_{y}(\omega)}{X^{0}(\omega)+E_{x}(\omega)},
$$

is approximated as

$$
H(\omega) \simeq \frac{Y^{o b s}(\omega)}{X^{0}(\omega)}\left(1-\frac{E_{y}(\omega)}{Y^{o b s}(\omega)}-\frac{E_{x}(\omega)}{X^{0}(\omega)}\right) .
$$

Then, it is further approximated as

$$
H(\omega) \simeq \frac{Y^{o b s}(\omega)-E_{y}(\omega)}{X^{0}(\omega)}
$$

with the condition of Eq. (5). This approximated system in the time domain is shown in Fig. 1(b). In this case, an input of the transfer function $h(n)$ is the accurately controlled transmitted signal $x^{0}(n)$, which is known. Because of this, we can define an observed transfer function $H^{o b s}(\omega)$ as

$$
H^{o b s}(\omega)=\frac{Y^{o b s}(\omega)}{X^{0}(\omega)} .
$$

and its observation noise as

$$
E_{h}(\omega)=\frac{E_{y}(\omega)}{X^{0}(\omega)}
$$

Then we have

$$
H^{o b s}(\omega)=1 \cdot H(\omega)+E_{h}(\omega)
$$

which is equivalent to a system model shown in Fig. 1(c).

The system of the transfer function $h(n)$ has an impulse input, which is an inverse Fourier transform of unity. The observation noise $\varepsilon_{h}(n)$ and the final output $h^{o b s}(n)$ are inverse Fourier transforms of $E_{h}(\omega)$ and $H^{o b s}(\omega)$, respectively. As shown in Eq. (12) the estimation of $h(n)$ by observation is a linear problem because the observable quantity $h^{o b s}(n)$ is the linear sum of unknown parameters $\varepsilon_{h}(n)$ and $h(n)$. Thus, the accurately controlled transmitted signal makes it possible to directly observe the transfer function $h^{o b s}(n)$ and adds the advantage of estimating the transfer function $h(n)$ as a linear problem. Such estimation can be performed more precisely than in the case of a non-linear problem, which should be solved as in the case of an ordinary controlled signal.

This precise estimation of the transfer function $h(n)$ with the accurately controlled signal provides us with better information on the underground structure.

\section{Description of the Transfer Function}

In order to impart a physical meaning to the transfer function in frequency domain, $H(\omega)$, we describe it with a ray model based on geometrical optics and Maxwell's equations. We consider a plane wave in an isotropic homogeneous medium. Though there are transfer functions for both electric and magnetic fields, we present only the case for an electric field. The magnetic field case can be easily obtained in a similar way (see Appendix A). 


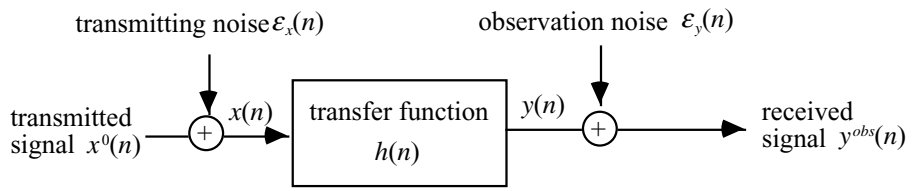

(a)

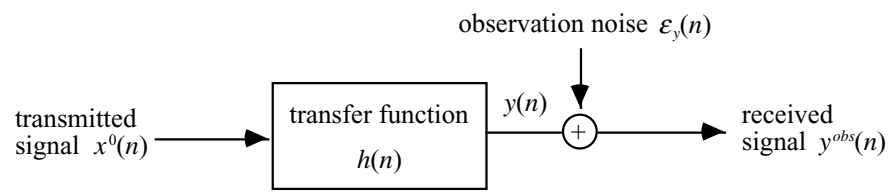

(b)

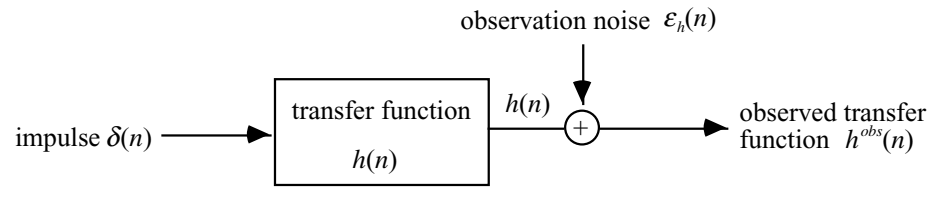

(c)

Fig. 1. Systems of the transmitted and received signals in the cases of (a) non-accurately controlled signal and (b) accurately controlled signal, and (c) the system equivalent to that of $(b)$.

\subsection{Ray model}

The electric field $\boldsymbol{E}$ in an isotropic homogeneous medium obeys the wave equation

$$
\mu \sigma \frac{\partial \boldsymbol{E}}{\partial t}+\mu \varepsilon \frac{\partial^{2} \boldsymbol{E}}{\partial t^{2}}=\nabla^{2} \boldsymbol{E}
$$

where $\mu, \sigma$, and $\varepsilon$ indicate magnetic permeability, electric conductivity, and dielectric permittivity, respectively. In the case of the plane wave, the solution of the wave equation becomes

$$
\boldsymbol{E}(\omega)=\boldsymbol{E}_{0} \exp i(\omega t-\boldsymbol{k}(\omega) \cdot \boldsymbol{r})
$$

where $\omega, \boldsymbol{k}(\omega)$, and $\boldsymbol{r}$, respectively, indicate angular frequency, wave number vector, and position vector. In order to make the electric field decay in the positive $r$ direction, the amplitude of the wave number becomes

$$
\begin{aligned}
k(\omega)= & \omega \sqrt{\frac{\mu \varepsilon}{2}\left(\sqrt{1+\left(\frac{\sigma}{\varepsilon \omega}\right)^{2}}+1\right)} \\
& -i \omega \sqrt{\frac{\mu \varepsilon}{2}\left(\sqrt{1+\left(\frac{\sigma}{\varepsilon \omega}\right)^{2}}-1\right)} .
\end{aligned}
$$

The first term of the right-hand side of Eq. (15) indicates phase delay, and the second term specifies amplitude attenuation. Even if the amplitude attenuation is large at low frequency, the first term never will be zero. That is, the electromagnetic field always propagates. Accordingly, we can use the concept of the wave front generally defined in physics and then describe the propagation of an electromagnetic field along rays as shown in Fig. 2(a). Summarizing, we can generally use the ray description in both cases of diffusing and non-diffusing electromagnetic fields.
In order to describe the whole transmitted electromagnetic field, which spreads in wide space, we need an infinite number of rays. Once the receiving point is fixed, only the rays that pass through the paths between the transmitting and receiving points are relevant to the measurement as shown in Fig. 2(b). The number of paths is limited because an electric field weaker than a threshold can be ignored in practice. Hence, we can simplify the description of the electromagnetic wave propagation with a finite number of paths. We shall call such a simple description a ray model and hereafter describe the transfer function with this model. Note that the model describes both the cases of diffusing and non-diffusing electromagnetic fields. That is, the model includes the case of a low-frequency region treated in MT and TEM as well as the case of a high-frequency region treated in GPR. We note that the ray model excludes the case involving resonance, for which an infinite number of rays is required.

\subsection{Three factors of the transfer function}

Let both the transmitted and received signals be electric vector fields. The transfer function defined in Eq. (9) becomes a second-rank tensor with nine elements:

$$
\boldsymbol{H}(\omega)=\left(\begin{array}{lll}
H_{x x}(\omega) & H_{x y}(\omega) & H_{x z}(\omega) \\
H_{y x}(\omega) & H_{y y}(\omega) & H_{y z}(\omega) \\
H_{z x}(\omega) & H_{z y}(\omega) & H_{z z}(\omega)
\end{array}\right)
$$

The transfer function includes three types of information: electromagnetic parameters, propagation distance, and boundary planes of the underground. In the following subsections, we derive the construction of the information.

According to the ray model, the transfer function becomes the sum of a finite number, $M$, of ray functions $\boldsymbol{H}^{m}(\omega)$ of 


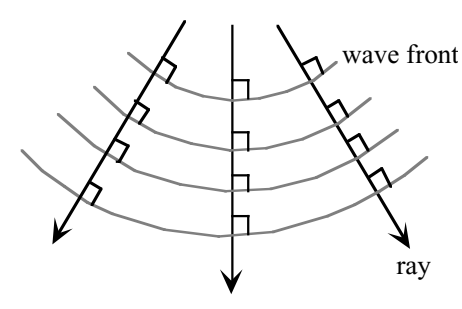

(a)

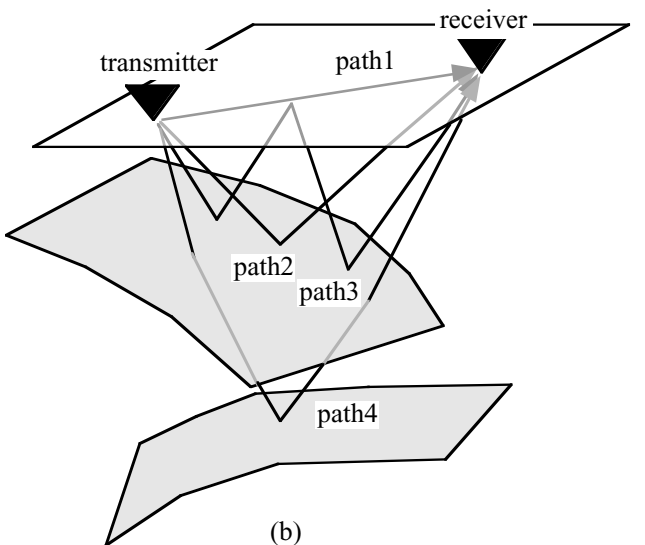

(b)

Fig. 2. (a) Rays and wave fronts, and (b) paths between the transmitting and receiving points.

path $m$ as

$$
\boldsymbol{H}(\omega)=\sum_{m=1}^{M} \boldsymbol{H}^{m}(\omega)
$$

The ray function is a product of the factors that indicate amplitude attenuation and phase delay accompanied by propagation in the homogeneous medium, together with reflection and refraction. Hence, we need to derive the factors concerned with propagation, reflection, and refraction to obtain the transfer function $\boldsymbol{H}(\omega)$.

We first derive the function of propagation $\boldsymbol{H}^{P}(\omega)$, which indicates amplitude attenuation and phase delay accompanied with propagation. This function $\boldsymbol{H}^{P}(\omega)$ in an isotropic homogeneous medium is independent of polarization of the electric field. Then it is written with an identity matrix $\mathbf{I}$ as

$$
\boldsymbol{H}^{P}(\omega)=H^{P}(\omega) \mathbf{I} .
$$

From Eq. (14), $H^{P}(\omega)$ becomes

$$
H^{P}(\omega)=\exp (-i \boldsymbol{k}(\omega) \cdot \boldsymbol{r})
$$

Next, we derive the function of reflection $\boldsymbol{H}^{R}(\omega)$, which indicates amplitude attenuation and phase delay induced by reflection. This function is calculated at a boundary plane between Medium 1 with parameters $\mu_{1}, \varepsilon_{1}$, and $\sigma_{1}$, and Medium 2 with parameters $\mu_{2}, \varepsilon_{2}$, and $\sigma_{2}$. The function of reflection consists of a rotation matrix and reflection coefficients. The rotation matrix $\boldsymbol{S}$ written by the form

$$
\boldsymbol{S}=\left(\begin{array}{lll}
s_{11} & s_{12} & s_{13} \\
s_{21} & s_{22} & s_{23} \\
s_{31} & s_{32} & s_{33}
\end{array}\right)
$$

and the elements satisfy the relations

$$
\begin{aligned}
s_{11}^{2}+s_{12}^{2}+s_{13}^{2} & =s_{21}^{2}+s_{22}^{2}+s_{23}^{2} \\
& =s_{31}^{2}+s_{32}^{2}+s_{33}^{2}=1
\end{aligned}
$$

and

$$
\begin{aligned}
s_{11} s_{21}+s_{12} s_{22}+s_{13} s_{23} & =s_{21} s_{31}+s_{22} s_{32}+s_{23} s_{33} \\
& =s_{31} s_{11}+s_{32} s_{12}+s_{33} s_{13} \\
& =0 .
\end{aligned}
$$

Therefore, the transpose and inverse of the matrix are equal:

$$
\boldsymbol{S}^{-1}=\boldsymbol{S}^{T} \text {. }
$$

This matrix transfers the firstly set coordinates $(x, y, z)$ to $\left(x^{\prime}, y^{\prime}, z^{\prime}\right)$ in order to set the ray on $x^{\prime} z^{\prime}$-plane and to set the boundary on $x^{\prime} y^{\prime}$-plane.

The reflection coefficients depends on the polarization of the electric field. The coefficients for $x^{\prime}, y^{\prime}$, and $z^{\prime}$ polarizations are (Ward and Hohmann, 1987)

$$
\begin{aligned}
& H_{x^{\prime}}^{R}(\omega)=\frac{-\mu_{1} k_{2} \cos \theta_{1}+\mu_{2} k_{1} \cos \theta_{2}}{\mu_{1} k_{2} \cos \theta_{1}+\mu_{2} k_{1} \cos \theta_{2}}, \\
& H_{y^{\prime}}^{R}(\omega)=\frac{-\mu_{1} k_{2} \cos \theta_{2}+\mu_{2} k_{1} \cos \theta_{1}}{\mu_{1} k_{2} \cos \theta_{2}+\mu_{2} k_{1} \cos \theta_{1}},
\end{aligned}
$$

and

$$
H_{z^{\prime}}^{R}(\omega)=-H_{x^{\prime}}^{R}(\omega)
$$

In Eqs. (24)-(26), angle of incidence $\theta_{1}$ and angle of refraction $\theta_{2}$ (complex when $\theta_{1}$ exceeds the critical angle) are related by

$$
\cos ^{2} \theta_{2}=1-\left(\frac{k_{1}}{k_{2}}\right)^{2} \sin ^{2} \theta_{1} .
$$

The wave number $k_{1}$ in Medium 1 and $k_{2}$ in Medium 2 are respectively defined as

$$
k_{1}^{2}=\mu_{1} \varepsilon_{1} \omega^{2}\left(1-i \frac{\sigma_{1}}{\varepsilon_{1} \omega}\right)
$$

and

$$
k_{2}^{2}=\mu_{2} \varepsilon_{2} \omega^{2}\left(1-i \frac{\sigma_{2}}{\varepsilon_{2} \omega}\right) .
$$

Here $x^{\prime} z^{\prime}$-polarization indicates a parallel incidence to the reflection plane and $y^{\prime}$-polarization indicates a normal incidence.

Combining the rotation and the reflection matrices, the function of the reflection $\boldsymbol{H}^{R}(\omega)$ becomes

$$
\boldsymbol{H}^{R}(\omega)=\boldsymbol{S}^{-1}\left(\begin{array}{ccc}
H_{x^{\prime}}^{R}(\omega) & 0 & 0 \\
0 & H_{y^{\prime}}^{R}(\omega) & 0 \\
0 & 0 & -H_{x^{\prime}}^{R}(\omega)
\end{array}\right) \boldsymbol{S}
$$


Substituting the elements of the rotation matrix into Eq. (30), we have

$$
\boldsymbol{H}^{R}(\omega)=H_{x^{\prime}}^{R}(\omega)(\boldsymbol{A}-\boldsymbol{C})+H_{y^{\prime}}^{R}(\omega) \boldsymbol{B}
$$

where

$$
\begin{aligned}
\boldsymbol{A} & =\left(\begin{array}{ccc}
s_{11}{ }^{2} & s_{11} s_{12} & s_{11} s_{13} \\
s_{11} s_{12} & s_{12}{ }^{2} & s_{12} s_{13} \\
s_{11} s_{13} & s_{12} s_{13} & s_{13}{ }^{2}
\end{array}\right), \\
\boldsymbol{B} & =\left(\begin{array}{ccc}
s_{21}{ }^{2} & s_{21} s_{22} & s_{21} s_{23} \\
s_{21} s_{22} & s_{22}{ }^{2} & s_{22} s_{23} \\
s_{21} s_{23} & s_{22} s_{23} & s_{23}{ }^{2}
\end{array}\right),
\end{aligned}
$$

and

$$
C=\left(\begin{array}{ccc}
s_{31}^{2} & s_{31} s_{32} & s_{31} s_{33} \\
s_{31} s_{32} & s_{32}{ }^{2} & s_{32} s_{33} \\
s_{31} s_{33} & s_{32} s_{33} & s_{33}^{2}
\end{array}\right)
$$

The function of the reflection $\boldsymbol{H}^{R}(\omega)$ consists of two complex scalar functions $H_{x^{\prime}}^{R}(\omega)$ and $H_{y^{\prime}}^{R}(\omega)$, and three real symmetric matrices $\boldsymbol{A}, \boldsymbol{B}$, and $\boldsymbol{C}$, which are independent of frequency. Hence, the elements of the tensor $\boldsymbol{H}^{R}(\omega)$ have common scalar functions $H_{x^{\prime}}^{R}(\omega)$ and $H_{y^{\prime}}^{R}(\omega)$ and different coefficients.

The function of refraction $\boldsymbol{H}^{T}(\omega)$, which indicates amplitude attenuation and phase delay accompanied by refraction, is derived in a similar way to that of reflection:

$$
\boldsymbol{H}^{T}(\omega)=\boldsymbol{S}^{-1}\left(\begin{array}{ccc}
H_{x^{\prime}}^{T}(\omega) & 0 & 0 \\
0 & H_{y^{\prime}}^{T}(\omega) & 0 \\
0 & 0 & H_{z^{\prime}}^{T}(\omega)
\end{array}\right) \boldsymbol{S}
$$

where

$$
\begin{aligned}
& H_{x^{\prime}}^{T}(\omega)=\frac{2 \mu_{2} k_{1} \cos \theta_{2}}{\mu_{1} k_{2} \cos \theta_{1}+\mu_{2} k_{1} \cos \theta_{2}}, \\
& H_{y^{\prime}}^{T}(\omega)=\frac{2 \mu_{2} k_{1} \cos \theta_{1}}{\mu_{1} k_{2} \cos \theta_{2}+\mu_{2} k_{1} \cos \theta_{1}},
\end{aligned}
$$

and

$$
H_{z^{\prime}}^{T}(\omega)=\frac{\tan \theta_{2}}{\tan \theta_{1}} H_{x}^{T}(\omega)
$$

Then, we have

$$
\boldsymbol{H}^{T}(\omega)=H_{x^{\prime}}^{T}(\omega) \boldsymbol{A}+H_{y^{\prime}}^{T}(\omega) \boldsymbol{B}+H_{z^{\prime}}^{T}(\omega) \boldsymbol{C} .
$$

Elements of the tensor function $\boldsymbol{H}^{T}(\omega)$ are linear combinations of common scalar functions $H_{x^{\prime}}^{T}(\omega), H_{y^{\prime}}^{T}(\omega)$, and $H_{z^{\prime}}^{T}(\omega)$ with different coefficients.

\subsection{Form of the transfer function}

We derived the three factors of the transfer function, the functions of propagation $\boldsymbol{H}^{P}(\omega)$, reflection $\boldsymbol{H}^{R}(\omega)$, and refraction $\boldsymbol{H}^{T}(\omega)$, in the previous subsection. Because the ray function of path $m, \boldsymbol{H}^{m}(\omega)$, is a product of the three factors, the ray function consists of complex scalar functions $F_{\ell}^{m}(\omega)$ and real coefficient matrices $\boldsymbol{G}_{\ell}^{m}$ as

$$
\boldsymbol{H}^{m}(\omega)=\sum_{\ell=1}^{L^{m}} \boldsymbol{G}_{\ell}^{m} F_{\ell}^{m}(\omega),
$$

where

$$
\begin{aligned}
F_{\ell}^{m}(\omega)= & \prod_{i=1}^{I} H^{P_{m i}}(\omega) \prod_{j=1}^{J}\left\{\begin{array}{l}
H_{x^{\prime}}^{R_{m j}}(\omega) \\
H_{y^{\prime}}^{R_{m j}}(\omega)
\end{array}\right\} \\
& \times \prod_{k=1}^{K}\left\{\begin{array}{l}
H_{x^{\prime}}^{T_{m k}}(\omega) \\
H_{y^{\prime}}^{T_{m k}}(\omega) \\
H_{z^{\prime}}^{T_{m k}}(\omega)
\end{array}\right\}
\end{aligned}
$$

and

$$
\boldsymbol{G}_{\ell}^{m}=\prod_{j=1}^{J}\left\{\begin{array}{c}
\boldsymbol{A}^{m j}-\boldsymbol{C}^{m j} \\
\boldsymbol{B}^{m j}
\end{array}\right\} \prod_{k=1}^{K}\left\{\begin{array}{l}
\boldsymbol{A}^{m k} \\
\boldsymbol{B}^{m k} \\
\boldsymbol{C}^{m k}
\end{array}\right\}
$$

In the above equations, \{\} indicates a selection operation. The symbols $I, J$, and $K$, respectively, indicate the number of the three following behaviors, propagation, reflection and refraction. A product of the matrices in Eq. (42) should be written in turn of occurrences of reflection and refraction, for example, reflection-reflection-reflection, or refractionreflection-refraction. However, a general expression of the turn is difficult, so that we write it as in Eq. (42) for convenience. From Eqs. (41) and (42), the number of $F_{\ell}^{m}(\omega)$ and $\boldsymbol{G}_{\ell}^{m}$ doubles when a reflection occurs and triples when a refraction occurs, so that

$$
L^{m}=2^{J} 3^{K} .
$$

Because the transfer function $\boldsymbol{H}(\omega)$ is a sum of the ray functions $\boldsymbol{H}^{m}(\omega)$, we finally have

$$
\boldsymbol{H}(\omega)=\sum_{m=1}^{M} \boldsymbol{H}^{m}(\omega)=\sum_{m=1}^{M} \sum_{\ell=1}^{L^{m}} \boldsymbol{G}_{\ell}^{m} F_{\ell}^{m}(\omega),
$$

where $M$ is the number of paths. Equation (44) is a form of the transfer function based on the ray model.

\subsection{Special cases}

The tensor transfer function $\boldsymbol{H}(\omega)$ derived as in Eq. (44) generally has nine elements. However, there are four cases when the transfer function does not have nine independent elements. The special cases are summarized in Table 1.

The first case is that of a homogeneous structure. The transfer function equals Eq. (18) in this case, and has one independent element.

The second and third cases are of a horizontal multi-layered structure. We set the coordinates to make the path on the $x z$-plane and to make the boundary layers parallel to the $x y$-plane. Hence, we can set the rotation matrix $\boldsymbol{S}$ as a unit matrix. Then the transfer function, which is a product of Eqs. (18), (30) and (35), becomes a diagonal matrix. Though the diagonal matrix generally has three different elements, it has only two elements when refraction does not occur.

The last case is a $3 \mathrm{D}$ structure, but reflection or refraction occurs only one time. In this case, the transfer function is a product of Eqs. (18) and (31) or Eqs. (18) and (39). Then the function has a symmetric form because matrices $\boldsymbol{A}, \boldsymbol{B}$, and $\boldsymbol{C}$ are symmetric.

\subsection{Structure of the transfer function}

We formulated the transfer function $\boldsymbol{H}(\omega)$ as in Eq. (44). Applying the derived form to the system in Fig. 1(c), an element of the tensor transfer function $\boldsymbol{H}(\omega)$ can be displayed 
Table 1. Independent elements and tensor form of the transfer function.

\begin{tabular}{|c|c|c|c|c|}
\hline structure & independent & \multicolumn{3}{|c|}{ matrix form } \\
\hline homogeneous & 1 & $\left(\begin{array}{c}H_{x x}(\omega) \\
0 \\
0\end{array}\right.$ & $\begin{array}{c}0 \\
H_{x x}(\omega) \\
0\end{array}$ & $\left.\begin{array}{c}0 \\
0 \\
H_{x x}(\omega)\end{array}\right)$ \\
\hline $\begin{array}{l}\text { horizontal mutilayers } \\
\text { (no refraction) }\end{array}$ & 2 & $\begin{array}{c}H_{x x}(\omega) \\
0 \\
0\end{array}$ & $\begin{array}{c}0 \\
H_{y y}(\omega) \\
0\end{array}$ & $\begin{array}{c}0 \\
0 \\
-H_{x x}(\omega)\end{array}$ \\
\hline $\begin{array}{l}\text { horizontal multilayers } \\
\text { (general) }\end{array}$ & 3 & $\left(\begin{array}{c}H_{x x}(\omega) \\
0 \\
0\end{array}\right.$ & $\begin{array}{c}0 \\
H_{y y}(\omega) \\
0\end{array}$ & $\left.\begin{array}{c}0 \\
0 \\
H_{z z}(\omega)\end{array}\right)$ \\
\hline $\begin{array}{l}3 \mathrm{D} \\
\text { (single reflection/refraction) }\end{array}$ & 6 & $\left(\begin{array}{l}H_{x x}(\omega) \\
H_{x y}(\omega) \\
H_{x z}(\omega)\end{array}\right.$ & $\begin{array}{l}H_{x y}(\omega) \\
H_{y y}(\omega) \\
H_{y z}(\omega)\end{array}$ & $\left.\begin{array}{c}H_{x z}(\omega) \\
H_{y z}(\omega) \\
H_{z z}(\omega)\end{array}\right)$ \\
\hline $\begin{array}{l}3 \mathrm{D} \\
\text { (general) }\end{array}$ & 9 & $\left(\begin{array}{l}H_{x x}(\omega) \\
H_{y x}(\omega) \\
H_{z x}(\omega)\end{array}\right.$ & $\begin{array}{l}H_{x y}(\omega) \\
H_{y y}(\omega) \\
H_{z y}(\omega)\end{array}$ & $\left.\begin{array}{c}H_{x z}(\omega) \\
H_{y z}(\omega) \\
H_{z z}(\omega)\end{array}\right)$ \\
\hline
\end{tabular}

as in Fig. 3. Here the system is initially expanded into $M$ branches of the paths, and then into $L^{m}$ branches of the polarizations. The system input is $F_{\ell}^{m}(\omega)$-operated and $\boldsymbol{G}_{\ell}^{m}$ amplified on each branch. The sum of the output from all branches with additional observation noise is the system output.

The first $M$ branching of the system is caused by the different electromagnetic structure where the ray passed through. The second $L^{m}$ branching is due to the different polarized directions of the electric field. The reflection coefficient is different for $x^{\prime} z^{\prime}$-polarization and $y^{\prime}$-polarization, so that the response includes two functions of $F_{\ell}^{m}(\omega)$. In the case of refraction, the response includes three functions of $F_{\ell}^{m}(\omega)$. The electric field changes its polarization when it is reflected and refracted, and a total combination of polarizations becomes $L^{m}$, as in Eq. (43).

On each branch, the input is operated with the complex function $F_{\ell}^{m}(\omega)$. The $F_{\ell}^{m}(\omega)$-operation leads amplitude attenuation and phase delay depending on the path $m$ and the polarization $\ell$. This attenuation and delay have information on electromagnetic parameters and propagation distance, because $F_{\ell}^{m}(\omega)$ consists of propagation function, reflection coefficients, and refraction coefficients. The output of $F_{\ell}^{m}(\omega)$-operation is amplified by the real coefficient $\boldsymbol{G}_{\ell}^{m}$. Because the coefficient $\boldsymbol{G}_{\ell}^{m}$ consists of the elements of the rotation matrix, it carries information of the boundary planes.

Summarizing, elements of the tensor transfer function $\boldsymbol{H}(\omega)$ consist of three types of information: electromagnetic parameters, propagation distance, and boundary planes.

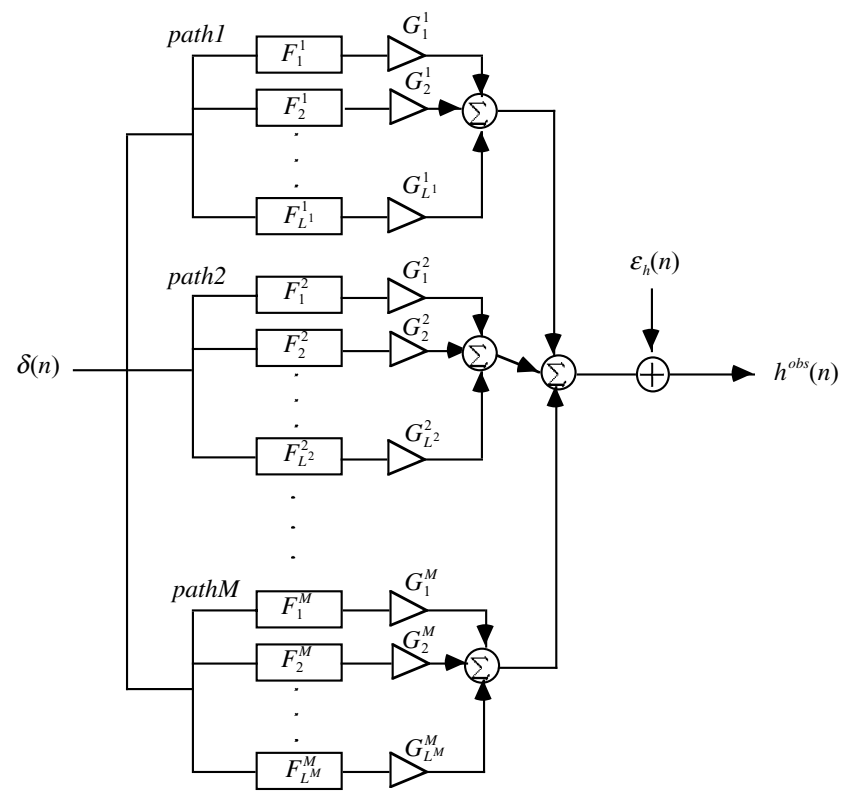

Fig. 3. Construction of physical information in the transfer function. The system is expanded into branches of the paths and then into branches of the polarization.

Among the types of information, that of electromagnetic parameters and distance is common to nine elements of the tensor. On the other hand, information of the boundary planes is included in a different form by the elements. 


\section{Numerical Examples}

We show simple numerical examples of the transfer function $\boldsymbol{H}(\omega)$ in this section. We calculated the transfer functions in three different frequency regions considering simple structures with three different scales.

\subsection{A structure example}

We consider the two-layer structure shown in Fig. 4. The transmitting and receiving points are separated by a distance $2 a$, and their mid-point is set as $O$. We define a coordinate system $(x, y, z)$ of the surface that sets the $x$-axis on a straight line between the transmitting and receiving points. The other horizontal axis is set as $y$, and the vertical axis is $z$.

The structure includes two layers. The upper layer has electromagnetic properties $\mu_{1}, \sigma_{1}$, and $\varepsilon_{1}$, and the lower layer has $\mu_{2}, \sigma_{2}$, and $\varepsilon_{2}$.

The boundary of the two layers consists of two planes. One of the planes is parallel to the $x y$-plane and at depth $d$. The other plane is inclined with angles $\xi$ and $\eta$. This plane is obtained by two rotations. The first rotation with angle $\xi$ is around the axis on the parallel plane. This axis is in the $x$-direction and at a distance $b$ from the normal line drawn from $O$ to the parallel plane. The coordinates $\left(x^{\prime}, y^{\prime}, z^{\prime}\right)$ on the rotated plane is set to include a normal line from $O$ on $y^{\prime} z^{\prime}$-plane. The second rotation with angle $\eta$ is around the $y^{\prime}$-axis. The coordinates of the secondary-rotated plane, the inclined plane, $\left(x^{\prime \prime}, y^{\prime \prime}, z^{\prime \prime}\right)$ is set to include a normal line from $O$ in $x^{\prime \prime} z^{\prime \prime}$-plane. Then, the relation between the coordinates $(x, y, z)$ and $\left(x^{\prime \prime}, y^{\prime \prime}, z^{\prime \prime}\right)$ is written as

$$
\left(\begin{array}{l}
x^{\prime \prime} \\
y^{\prime \prime} \\
z^{\prime \prime}
\end{array}\right)=\boldsymbol{S}\left(\begin{array}{l}
x \\
y \\
z
\end{array}\right)
$$

where $\boldsymbol{S}$ is the transformation matrix of coordinates

$$
\boldsymbol{S}=\left(\begin{array}{ccc}
\cos \eta & -\sin \eta \sin \xi & \sin \eta \cos \xi \\
0 & \cos \xi & \sin \xi \\
-\sin \eta & -\cos \eta \sin \xi & \cos \eta \cos \xi
\end{array}\right)
$$

For the following calculation, we set three scale parameters $a, b$, and $d$ as $a=b=d$ for simplicity. We calculated the transfer function of three different cases: the scales of the structure are $10 \mathrm{~km}, 100 \mathrm{~m}$, and $50 \mathrm{~m}$. Angles of the inclined plane and the electromagnetic parameters are set as shown in Table 2.

\subsection{Paths}

We consider three paths in the example cases. Though we should consider more paths of multi-reflection in general, here, for simplicity, we consider the paths of single reflection, Path 1 connects the transmitting and receiving points with a straight line. Paths 2 and 3 connect the points of reflection on the parallel plane and the inclined plane, respectively.

For the first step to obtain the total transfer function $\boldsymbol{H}(\omega)$, we calculate the ray functions $\boldsymbol{H}^{m}(\omega)$. The function of Path $1, \boldsymbol{H}^{1}(\omega)$ is that of a propagation distance of $2 a$, so that we have

$$
\boldsymbol{H}^{1}(\omega)=\boldsymbol{H}^{P_{1}}(\omega)=H^{P_{1}}(\omega) \mathbf{I},
$$

Table 2. Structure parameters.

\begin{tabular}{cc}
\hline symbol & value \\
\hline$\xi$ & $10^{\circ}$ \\
$\eta$ & $30^{\circ}$ \\
$\mu_{1}$ & $\mu_{0}$ \\
$\sigma_{1}$ & $10^{-3} \mathrm{~S} / \mathrm{m}$ \\
$\varepsilon_{1}$ & $10 \varepsilon_{0}$ \\
$\mu_{2}$ & $\mu_{0}$ \\
$\sigma_{2}$ & $10^{-1} \mathrm{~S} / \mathrm{m}$ \\
$\varepsilon_{2}$ & $\varepsilon_{0}$ \\
\hline$\mu_{0}:$ permeability in vacuum. \\
$\varepsilon_{0}:$ permittivity in vacuum.
\end{tabular}

where

$$
H^{P_{1}}(\omega)=\exp \left(-2 i k_{1}(\omega) a\right) .
$$

Next, we calculate the function of Path $2, \boldsymbol{H}^{2}(\omega)$. This function consists of operations of propagation with distance $s_{1}$, reflection with angle of incidence $\theta$, and again propagation with distance $s_{1}$. Then, we have

$$
\begin{aligned}
\boldsymbol{H}^{2}(\omega)= & \boldsymbol{H}^{P_{2}}(\omega) \boldsymbol{H}^{R_{2}}(\omega) \boldsymbol{H}^{P_{2}}(\omega)=\left(H^{P_{2}}(\omega)\right)^{2} \\
& \times\left(\begin{array}{ccc}
H_{x}^{R_{2}}(\omega) & 0 & 0 \\
0 & H_{y}^{R_{2}}(\omega) & 0 \\
0 & 0 & -H_{x}^{R_{2}}(\omega)
\end{array}\right),
\end{aligned}
$$

where

$$
H^{P_{2}}(\omega)=\exp \left(-i k_{1}(\omega) s_{1}\right)
$$

and

$$
s_{1}=\sqrt{a^{2}+d^{2}} .
$$

The functions $H_{x}^{R_{2}}(\omega)$ and $H_{y}^{R_{2}}(\omega)$ are calculated from Eqs. (24) and (25) using

$$
\theta=\tan ^{-1} \frac{a}{d} .
$$

The function of the last Path $3 \boldsymbol{H}^{3}(\omega)$ consists of operations of propagation with distance $s_{1}^{\prime \prime}$, reflection with angle of incidence $\theta^{\prime \prime}$, and propagation with distance $s_{2}^{\prime \prime}$. Then we have

$$
\begin{aligned}
\boldsymbol{H}^{3}(\omega)= & \boldsymbol{H}^{P_{31}}(\omega) \boldsymbol{H}^{R_{3}}(\omega) \boldsymbol{H}^{P_{32}}(\omega) \\
= & H^{P_{31}}(\omega) H^{P_{32}}(\omega) \\
& \times \boldsymbol{S}^{-1}\left(\begin{array}{ccc}
H_{x^{\prime \prime}}^{R_{3}}(\omega) & 0 & 0 \\
0 & H_{y^{\prime \prime}}^{R_{3}}(\omega) & 0 \\
0 & 0 & -H_{x^{\prime \prime}}^{R_{3}}(\omega)
\end{array}\right) \boldsymbol{S},
\end{aligned}
$$

where

$$
\begin{gathered}
H^{P_{31}}(\omega)=\exp \left(-i k_{1}(\omega) s_{1}^{\prime \prime}\right), \\
H^{P_{32}}(\omega)=\exp \left(-i k_{1}(\omega) s_{2}^{\prime \prime}\right), \\
s_{1}^{\prime \prime}=\frac{\cos \eta}{\cos \theta^{\prime \prime}}(d \cos \xi+b \sin \xi-a \tan \eta),
\end{gathered}
$$




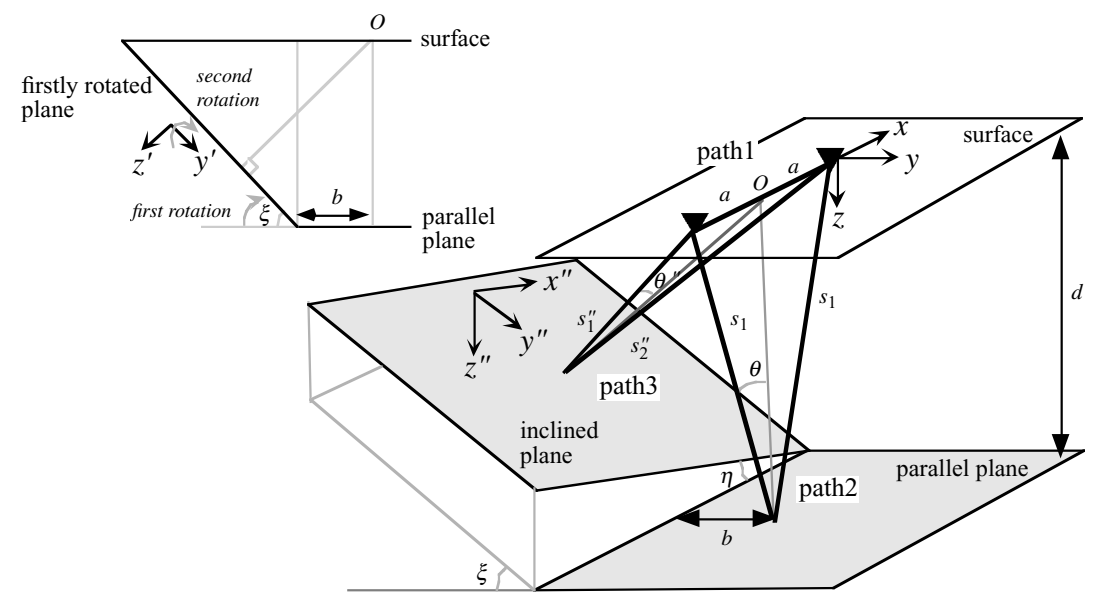

Fig. 4. An example for two layer structure. The boundary consists of two planes: one is pararell to the $x y$-plane and the other is inclined with angles $\xi$ and $\eta$.

Table 3. Parameters of propagation and reflection.

\begin{tabular}{|c|c|c|c|c|c|c|c|c|}
\hline \multirow{2}{*}{$\begin{array}{l}\text { frequency region } \\
\text { (frequency, scale) }\end{array}$} & \multirow[t]{2}{*}{ path } & \multirow{2}{*}{$\begin{array}{l}\text { propagation } \\
\text { distance }\end{array}$} & \multirow{2}{*}{$\begin{array}{l}\text { reflection } \\
\text { angle }\end{array}$} & \multicolumn{4}{|c|}{ reflection coefficient } & \multirow[t]{2}{*}{ cycle } \\
\hline & & & & $\left|H_{x}^{R}\right|$ & $\arg H_{x}^{R}$ & $\left|H_{y}^{R}\right|$ & $\arg H_{y}^{R}$ & \\
\hline low & Path 1 & $20 \mathrm{~km}$ & - & - & - & - & - & $15 \mathrm{~Hz}$ \\
\hline \multirow[t]{2}{*}{$(15 \mathrm{~Hz}, 10 \mathrm{~km})$} & Path 2 & $28 \mathrm{~km}$ & $45^{\circ}$ & 0.75 & $180^{\circ}$ & 0.77 & $180^{\circ}$ & $11 \mathrm{~Hz}$ \\
\hline & Path 3 & $23 \mathrm{~km}$ & $41^{\circ}$ & 0.87 & $180^{\circ}$ & 0.86 & $180^{\circ}$ & $13 \mathrm{~Hz}$ \\
\hline intermediate & Path 1 & $200 \mathrm{~m}$ & - & - & - & - & - & $290 \mathrm{kHz}$ \\
\hline \multirow[t]{2}{*}{$(600 \mathrm{kHz}, 100 \mathrm{~m})$} & Path 2 & $280 \mathrm{~m}$ & $45^{\circ}$ & 0.75 & $180^{\circ}$ & 0.76 & $180^{\circ}$ & $200 \mathrm{kHz}$ \\
\hline & Path 3 & $230 \mathrm{~m}$ & $41^{\circ}$ & 0.87 & $180^{\circ}$ & 0.86 & $180^{\circ}$ & $250 \mathrm{kHz}$ \\
\hline high & Path 1 & $100 \mathrm{~m}$ & - & - & - & - & - & $1.0 \mathrm{MHz}$ \\
\hline \multirow[t]{2}{*}{$(8.0 \mathrm{MHz}, 50 \mathrm{~m})$} & Path 2 & $140 \mathrm{~m}$ & $45^{\circ}$ & 0.63 & $160^{\circ}$ & 0.65 & $160^{\circ}$ & $0.73 \mathrm{MHz}$ \\
\hline & Path 3 & $120 \mathrm{~m}$ & $41^{\circ}$ & 0.79 & $170^{\circ}$ & 0.78 & $170^{\circ}$ & $0.89 \mathrm{MHz}$ \\
\hline
\end{tabular}

and

$$
s_{2}^{\prime \prime}=s_{1}^{\prime \prime}+\frac{2 a \sin \eta}{\cos \theta^{\prime \prime}} .
$$

In this case, the functions $H_{x^{\prime \prime}}^{R_{3}}(\omega)$ and $H_{y^{\prime \prime}}^{R_{3}}(\omega)$ are, respectively, the same to $H_{x}^{R_{2}}(\omega)$ and $H_{y}^{R_{2}}(\omega)$, but

$$
\theta^{\prime \prime}=\tan ^{-1} \frac{a}{d \cos \xi+b \sin \xi} .
$$

The transfer function $\boldsymbol{H}(\omega)$ is calculated from Eqs. (47), (49), and (53) as

$$
\boldsymbol{H}(\omega)=\boldsymbol{H}^{1}(\omega)+\boldsymbol{H}^{2}(\omega)+\mathbf{H}^{3}(\omega) .
$$

This tensor is symmetric and has six independent elements as shown in Table 1, because multi-reflection is not considered.

\subsection{The ray functions and the transfer functions}

Using the structure and the ray model mentioned above, we present numerical examples in three cases. We considered low-, intermediate-, and high-frequency regions represented by ranges of $1-30 \mathrm{~Hz}, 200 \mathrm{kHz}-1 \mathrm{MHz}$, and 6-10 $\mathrm{MHz}$, respectively. Then, we combined the ranges, respectively, with the $10 \mathrm{~km}, 100 \mathrm{~m}$, and $50 \mathrm{~m}$ scale structures.
The calculated ray functions $\boldsymbol{H}^{m}(\omega)$ in the case of low frequency and large scale are shown in Fig. 5(a). In order to help in the interpretation of the result, parameters of propagation and reflection at the central frequency of the range are given in Table 3. All the elements of the ray functions rapidly decay as frequency increases, because the attenuation increases as frequency increases. Because the tensor function of Path 1 indicates propagation in a homogeneous medium, it has an independent diagonal element, as shown in Table 1. The tensor function of Path 2 includes reflection on the parallel plane and has two independent diagonal elements. The tensor function of Path 3 is symmetric because it includes reflection on the inclined plane. Such reflections accompany phase reversal with the parameter in Table 2 , so that $H_{x x}^{m}$ of Paths 2 and 3 has mostly opposite signs to that of Path 1. Smaller amplitudes of the functions of Paths 2 and 3 are the results of the reflections and also of the longer propagation distances.

The tensor transfer function $\boldsymbol{H}(\omega)$ calculated from the ray functions is shown in Fig. 5(b). The diagonal elements of the tensor transfer function are sums of the diagonal elements of the three ray functions $\boldsymbol{H}^{m}(\omega)$. Concerning $H_{x x}$ and $H_{y y}$, amplitudes of the elements are smaller than those of Path 1. This is because of the opposite signs of the elements of 

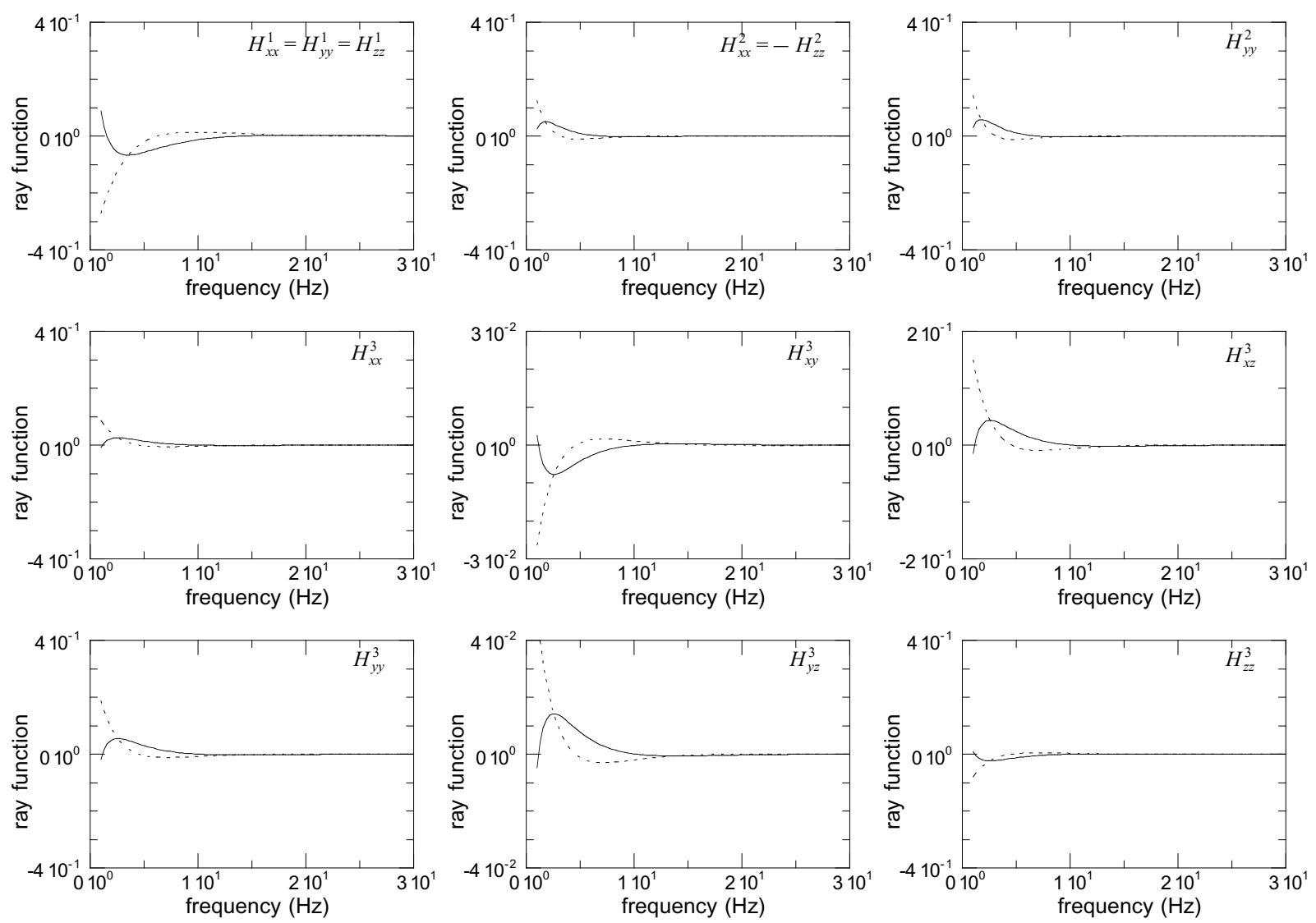

(a)
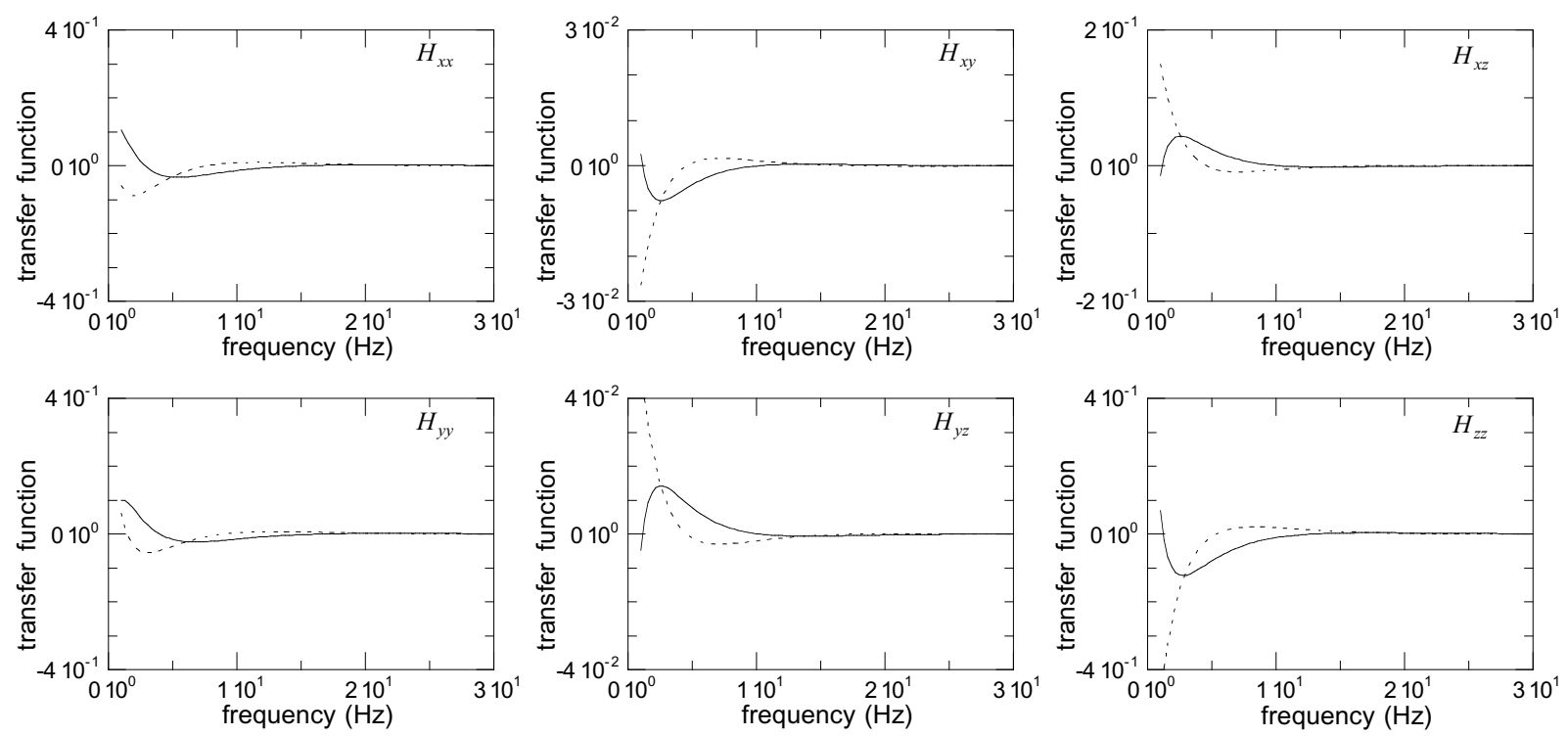

(b)

Fig. 5. Numerical example of (a) the ray functions and (b) the transfer function in the low-frequency region. Solid and broken lines indicate a real part and an imaginary part, respectively.

Paths 2 and 3 to those of Path 1. On the other hand, $H_{z z}^{2}$ has mostly the same signs to $H_{z z}^{\prime}$, so that $H_{z z}$ of the transfer function is larger than the element of Path 1. Off-diagonal elements of the transfer function are equal to those of Path 3. Because there is no contribution from the function of the shortest Path 1, the off-diagonal elements are smaller than diagonal elements.

When we observe such a transfer function, we can learn about the underground only from the outline of the function. Firstly, single reflection is known from the symmetry of the tensor function. Secondly, the 3D underground structure is known from the existence of the off-diagonal elements. 

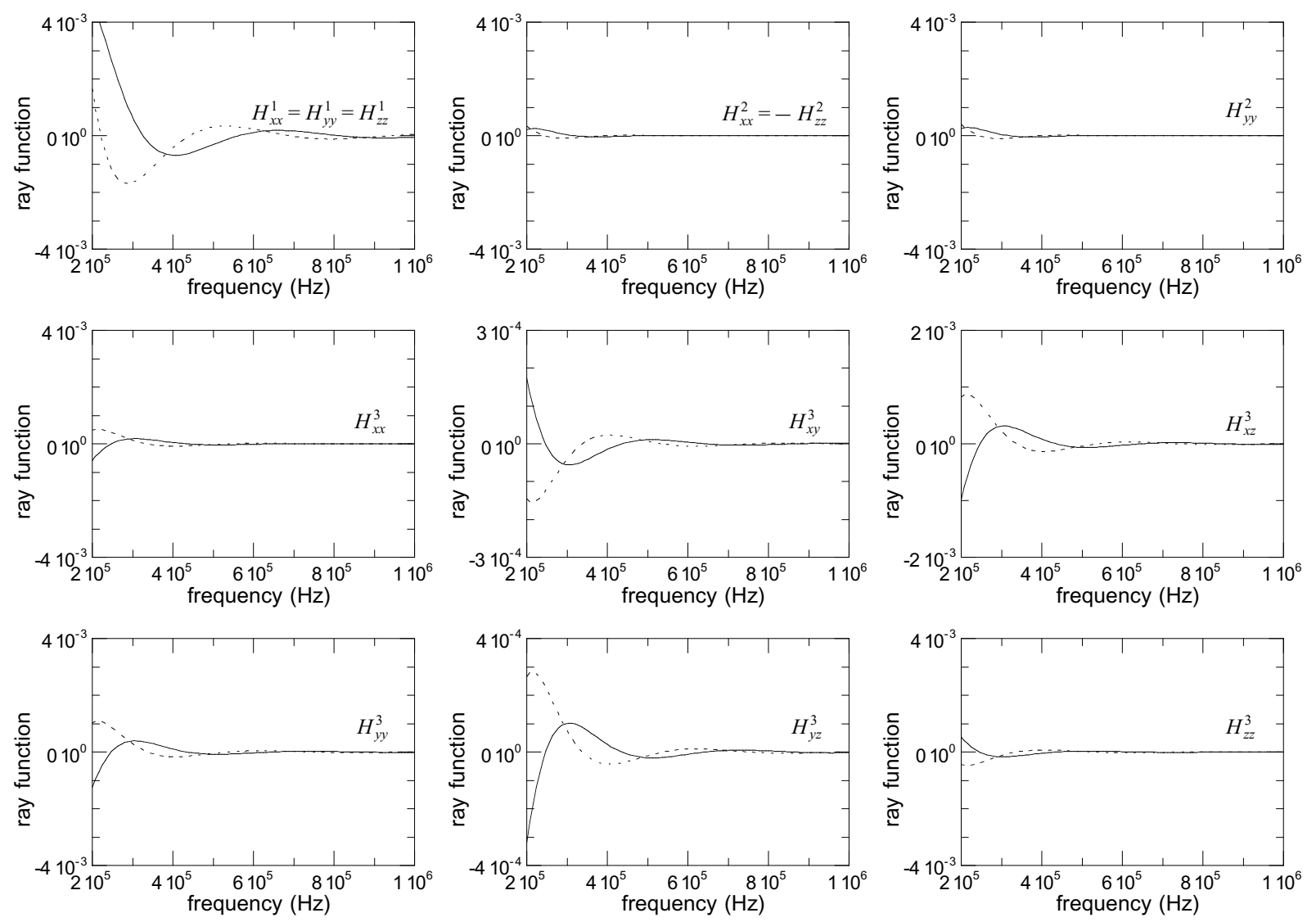

(a)
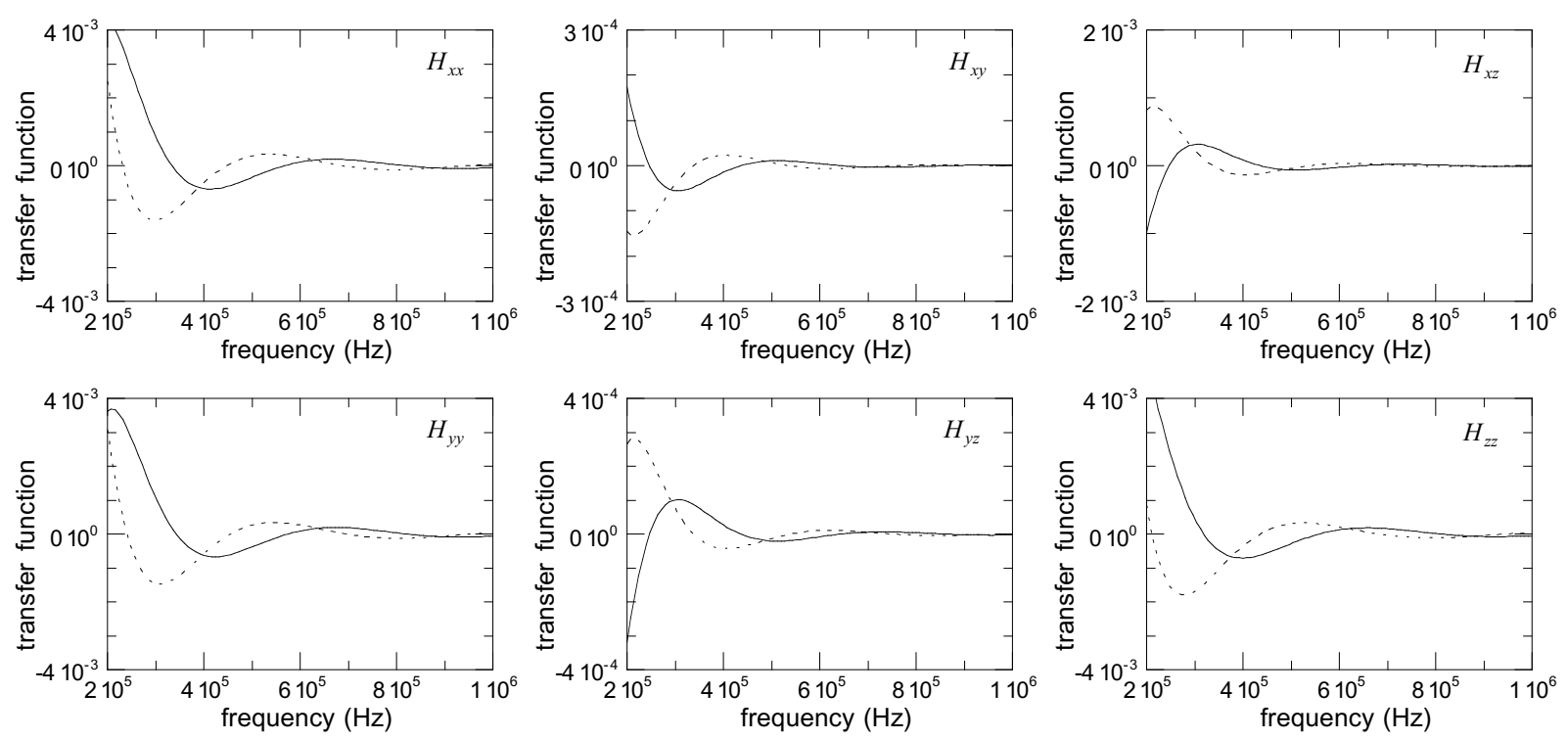

(b)

Fig. 6. Numerical example of (a) the ray functions and (b) the transfer function in the intermediate frequency region. Solid and broken lines indicate a real part and an imaginary part, respectively.

Lastly, an order of magnitude estimation of the depth of the reflection point is known from the differences between the diagonal elements.

We show the ray functions $\boldsymbol{H}^{m}(\omega)$ and the transfer function $\boldsymbol{H}(\omega)$ in the case of intermediate frequency and middlescale structure in Fig. 6. In this case, the decay of the func- tions is slow and an oscillatory tendency appears. The relation between the three ray functions and that between the elements of the tensor transfer function are similar to the first case.

We show the case of high frequency and small-scale structure in Fig. 7. The ray functions in this case scarcely decay, 

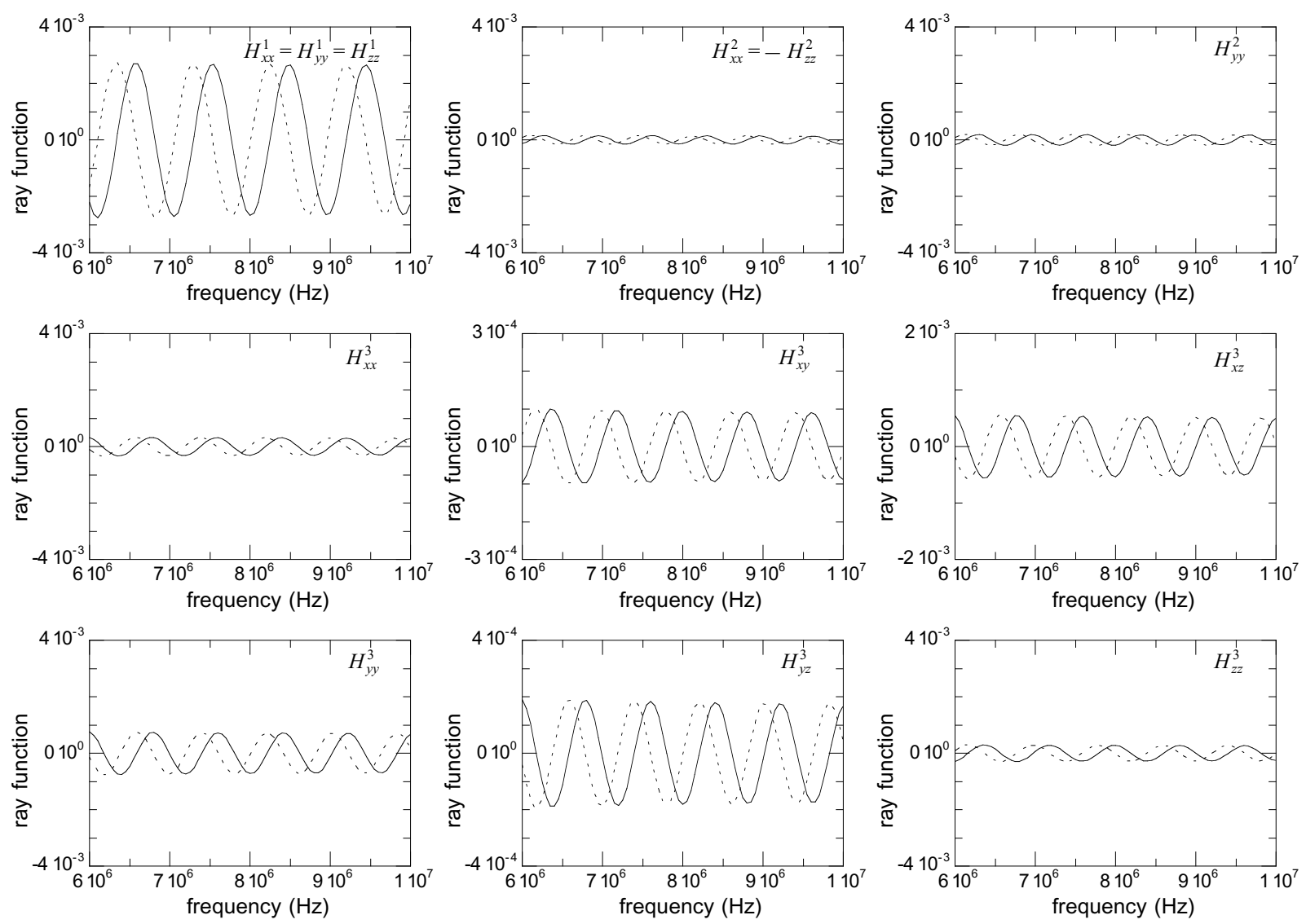

(a)
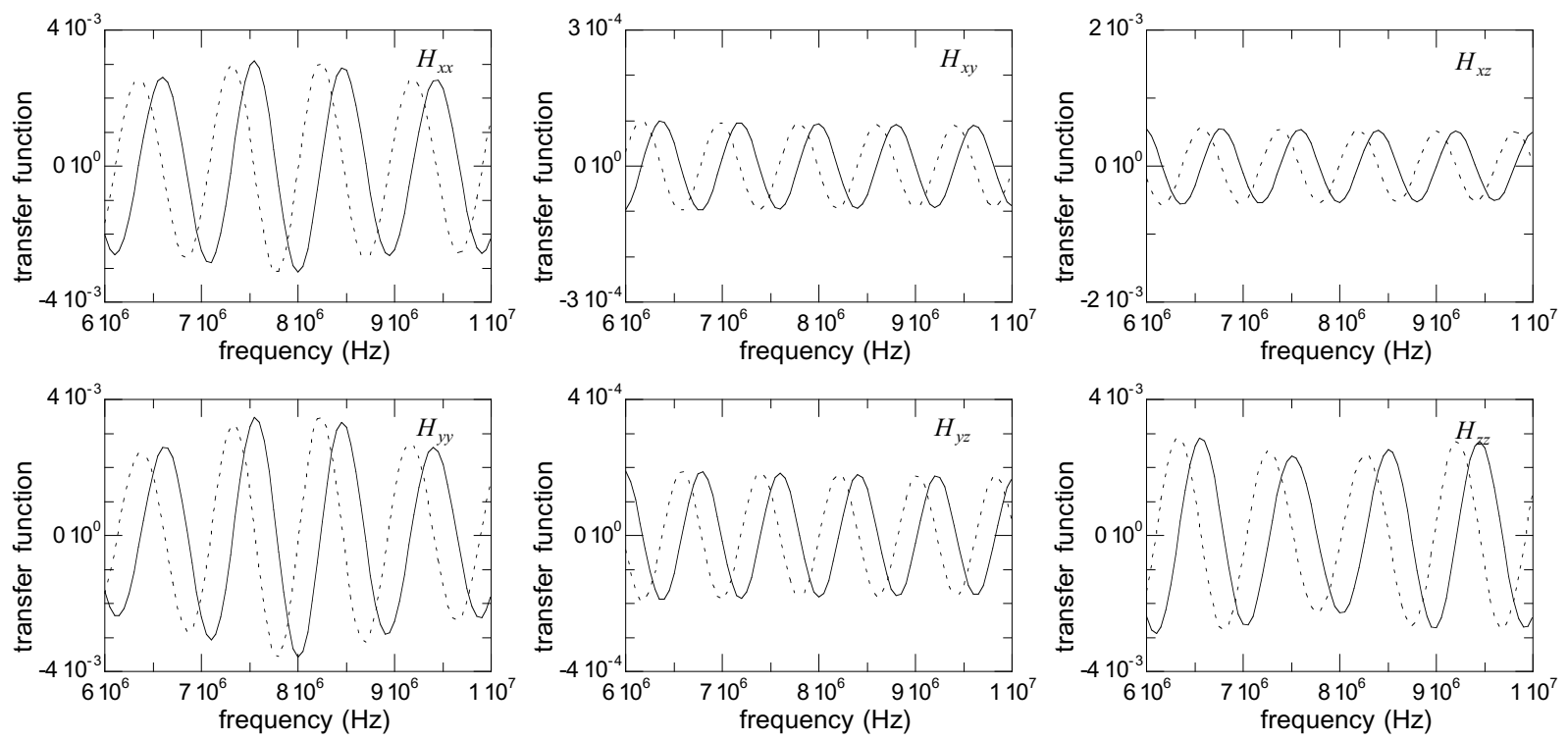

(b)

Fig. 7. Numerical example of (a) the ray functions and (b) the transfer function in the high frequency region. Solid and broken lines indicate a real part and an imaginary part, respectively.

but they do oscillate. The cycle of the oscillation is slightly different for the ray functions shown in Table 3. Such difference of the cycle also exists in the previous cases, though it is not clear because of the decay with frequency. The cycle of the oscillation roughly concerns group delay, which we explain in the following section. The relation of amplitudes among the ray function $\boldsymbol{H}^{m}(\omega)$ is mostly the same as the previous two cases. Concerning the transfer function $\boldsymbol{H}(\omega)$, dependency of the amplitude on frequency is different from the previous cases: amplitudes of the diagonal elements of the transfer function change with frequency. This amplitude modulation is a part of a beat synthesized from the ray func- 
tions with different cycles.

The typical profile of the transfer function is thus dependent on frequency. This is explained by the character of the medium. It acts as a conductor in low frequency and as a dielectric in the high frequency region.

\section{Approximated Model of the Transfer Function}

In the previous section, we showed that the profiles of the transfer functions $\boldsymbol{H}(\omega)$ are different throughout frequency regions. In this section, we represent the profiles in a narrow band with a simple function to improve our understanding.

The frequency function $F_{\ell}^{m}(\omega)$ has information of electromagnetic properties and propagation distance as mentioned above. From another viewpoint, the function indicates amplitude attenuation and phase delay. Describing the attenuation and the delay together, $F_{\ell}^{m}(\omega)$ is a function of complex attenuation. This function is related to the function of complex phase delay $\phi_{\ell}^{m}(\omega)$ as

$$
F_{\ell}^{m}(\omega)=\exp i \phi_{\ell}^{m}(\omega)
$$

Here we consider a linearization of the frequency function $F_{\ell}^{m}(\omega)$ or $\phi_{\ell}^{m}(\omega)$ to retain the physical meaning. Comparing linearization errors of cases $F_{\ell}^{m}(\omega)$ and $\phi_{\ell}^{m}(\omega)$, the approximation is valid in the wider frequency range in the case of $\phi_{\ell}^{m}(\omega)$ (see Appendix B for proof). Hence, we adopted a linearization of $\phi_{\ell}^{m}(\omega)$.

With Taylor expansion of $\phi_{\ell}^{m}(\omega)$ around a center angular frequency $\omega_{0}$, we have

$$
\phi_{\ell}^{m}(\omega) \simeq \phi_{\ell}^{m}\left(\omega_{0}\right)+\dot{\phi}_{\ell}^{m}\left(\omega_{0}\right)\left(\omega-\omega_{0}\right),
$$

defining

$$
\dot{\phi}_{\ell}^{m}\left(\omega_{0}\right)=\left.\frac{\mathrm{d} \phi_{\ell}^{m}(\omega)}{\mathrm{d} \omega}\right|_{\omega=\omega_{0}} .
$$

Substituting Eq. (61) into Eq. (60), we have

$$
F_{\ell}^{m}(\omega) \simeq F_{\ell}^{m}\left(\omega_{0}\right) \exp \left\{i \dot{\phi}_{\ell}^{m}\left(\omega_{0}\right)\left(\omega-\omega_{0}\right)\right\} .
$$

Then the transfer function in Eq. (44) becomes

$$
\begin{aligned}
\boldsymbol{H}(\omega) & \simeq \sum_{m=1}^{M} \sum_{\ell=1}^{L^{m}} \boldsymbol{G}_{\ell}^{m} F_{\ell}^{m}\left(\omega_{0}\right) \exp \left\{i \dot{\phi}_{\ell}^{m}\left(\omega_{0}\right)\left(\omega-\omega_{0}\right)\right\} \\
& =\sum_{m=1}^{M} \sum_{\ell=1}^{L^{m}} \boldsymbol{\beta}_{\ell}^{m} \exp \left\{i \alpha_{\ell}^{m}\left(\omega-\omega_{0}\right)\right\}
\end{aligned}
$$

defining

$$
\alpha_{\ell}^{m}=\dot{\phi}_{\ell}^{m}\left(\omega_{0}\right)
$$

and

$$
\boldsymbol{\beta}_{\ell}^{m}=\boldsymbol{G}_{\ell}^{m} F_{\ell}^{m}\left(\omega_{0}\right)
$$

The simplified transfer function in Eq. (64) is a linear combination of exponential functions $\mathrm{e}^{i \alpha_{\ell}^{m} \omega}$, whose amplitude is $\beta_{\ell}^{m}$ and initial angular frequency is $\omega_{0}$. The value $F_{\ell}^{m}\left(\omega_{0}\right)$ indicates amplitude attenuation and phase delay at $\omega_{0}$. The exponential function is generally a decaying or growing oscillation because $\alpha_{\ell}^{m}$ is generally complex. A cycle of the oscillation is $2 \pi / \operatorname{Re} \alpha_{\ell}^{m}$, where the real part of $\alpha_{\ell}^{m}$ indicates group delay at $\omega_{0}$ from the definition in Eq. (65). The imaginary part of $\alpha_{\ell}^{m}$ concerns an attenuation of the wave packet.

We understand the typical frequency profile of the transfer function with the approximated form. The rapidly decaying part of the transfer function in the low-frequency region can be approximated with a sum of real exponential functions, because the imaginary part is dominant in $\alpha_{\ell}^{m}$. On the other hand, the real part is dominant in the high-frequency region, and the transfer function can be approximated with a sum of sine curves. In the intermediate-frequency region, the profile is that of complex exponential functions. Such profiles are also recognized in the examples in Figs. 5 to 7.

\section{Discussion and Conclusions}

We pointed out that the transfer function between transmitted and received electromagnetic fields is observable when an accurately controlled signal is used. We also derived a form of transfer function based on the ray model. From the result, physical properties and the construction of physical information concerning the transfer function were clarified. The transfer function is not influenced by the transmitting signal but is a straight forward information of the underground structure. In other words, measurement of the transfer function is regard as direct measurement of the underground structure, and this measurement becomes possible by using an accurately controlled signal.

Such a measurement provides us with the potential to estimate information of an underground structure without noise as a linear inversion problem. This is in direct contrast to the methods with non-accurately controlled signals. In the latter cases, only the received electromagnetic field is observable and the transfer function cannot be measured. The unknown transmitting noise is convoluted in the observed signal, so that an estimation of information of the underground structure becomes a non-linear problem. On the other hand, the accurately controlled signal provides information on underground structures without involving a non-linear inversion problem. This is an advantage of using the accurately controlled signal in addition to the technical advantage of obtaining high SNR data (Nakajima et al., 2000).

We also approximated the transfer function with simple functions. It helped us to understand a rough frequency profile of the transfer function in a narrow band. It is also useful to make a plan of data processing. In order to estimate the underground structure, we remove noise and process the data before the estimation of the structure. In this process, the structure estimation benefits from the decomposition of the data into components with physical meaning. Hence, we can adopt an approximated transfer function, whose physical meaning is clarified, as mentioned in the previous section, as the decomposition model.

The approximated transfer function in Eq. (64) with observed noise can be reduced to an autoregressive (AR)-type model of the extended Prony method or Sompi method (Kay and Marple, 1981; Kumazawa et al., 1990). These methods are useful in decomposing the transfer function with weak decay, such as in Fig. 7. The decomposition was initially 
tried for an analysis of seismological data by Hasada et al. (2001), although their algorithm is not suitable to decompose the electromagnetic data. We developed a useful algorithm and succeeded in the decomposition of the transfer function in the case of two horizontal layers (Yokoyama et $a l .$, 2000a). An estimation of the structure using the decomposed components was also successful. From this experience, the decomposition seems to be also useful for an estimation of general 3D structure. Development of an algorithm that works in the case of strong decay, such as in the cases of Figs. 5 and 6, is an unresolved problem to realize a decomposition method.

Acknowledgments. We thank Dr. P. Weidelt for reviewing the manuscript critically.

\section{Appendix A.}

The transfer function of a magnetic field can be derived in a similar way to that shown in Section 3. But, $H_{x^{\prime}}^{R}(\omega)$, $H_{y^{\prime}}^{R}(\omega), H_{z^{\prime}}^{R}(\omega), H_{x^{\prime}}^{T}(\omega), H_{y^{\prime}}^{T}(\omega)$, and $H_{z^{\prime}}^{T}(\omega)$ in Eqs. $(24)-$ (26) and Eqs. (36)-(38) should be replaced by

$$
\begin{array}{r}
H_{x^{\prime}}^{R}(\omega)=\frac{\mu_{1} k_{2} \cos \theta_{2}-\mu_{2} k_{1} \cos \theta_{1}}{\mu_{1} k_{2} \cos \theta_{2}+\mu_{2} k_{1} \cos \theta_{1}}, \\
H_{y^{\prime}}^{R}(\omega)=\frac{\mu_{1} k_{2} \cos \theta_{1}-\mu_{2} k_{1} \cos \theta_{2}}{\mu_{1} k_{2} \cos \theta_{1}+\mu_{2} k_{1} \cos \theta_{2}}, \\
H_{z^{\prime}}^{R}(\omega)=-H_{x^{\prime}}^{R}(\omega), \\
H_{x^{\prime}}^{T}(\omega)=\frac{2 \mu_{1} k_{2} \cos \theta_{2}}{\mu_{1} k_{2} \cos \theta_{2}+\mu_{2} k_{1} \cos \theta_{1}}, \\
H_{y^{\prime}}^{T}(\omega)=\frac{2 \mu_{1} k_{2} \cos \theta_{1}}{\mu_{1} k_{2} \cos \theta_{1}+\mu_{2} k_{1} \cos \theta_{2}},
\end{array}
$$

and

$$
H_{z^{\prime}}^{T}(\omega)=\frac{\tan \theta_{2}}{\tan \theta_{1}} H_{x^{\prime}}^{T}(\omega) .
$$

\section{Appendix B.}

We compare linearization errors of the functions $F_{\ell}^{m}(\omega)$ and $\phi_{\ell}^{m}(\omega)$ in Eq. (60). Setting the angular frequency range as $\Delta \omega_{1}$, the error of the function $F_{\ell}^{m}(\omega)$ becomes

$$
\Delta F_{\ell}^{m}(\omega)=F_{\ell}^{m}(\omega)-\left(F_{\ell}^{m}\left(\omega_{0}\right)+\dot{F}_{\ell}^{m}\left(\omega_{0}\right) \Delta \omega_{1}\right) .
$$

On the other hand, the error of the function $\phi_{\ell}^{m}(\omega)$ in the range $\Delta \omega_{2}$ becomes

$$
\Delta F_{\ell}^{m}(\omega)=F_{\ell}^{m}(\omega)-F_{\ell}^{m}\left(\omega_{0}\right) \exp i \dot{\phi}_{\ell}^{m}\left(\omega_{0}\right) \Delta \omega_{2} .
$$

Setting both errors at the same level, we have

$$
\begin{aligned}
& F_{\ell}^{m}\left(\omega_{0}\right)+\dot{F}_{\ell}^{m}\left(\omega_{0}\right) \Delta \omega_{1} \\
& =F_{\ell}^{m}\left(\omega_{0}\right) \exp i \dot{\phi}_{\ell}^{m}\left(\omega_{0}\right) \Delta \omega_{2} .
\end{aligned}
$$

From the above equation, the ratio of the two frequency ranges becomes

$$
\begin{aligned}
\frac{\Delta \omega_{1}}{\Delta \omega_{2}} & =\frac{F_{\ell}^{m}\left(\omega_{0}\right) \exp i \dot{\phi}_{\ell}^{m}\left(\omega_{0}\right) \Delta \omega_{2}-F_{\ell}^{m}\left(\omega_{0}\right)}{\dot{F}_{\ell}^{m}\left(\omega_{0}\right) \Delta \omega_{2}} \\
& =\frac{\exp i \dot{\phi}_{\ell}^{m}\left(\omega_{0}\right) \Delta \omega_{2}-1}{i \dot{\phi}_{\ell}^{m}\left(\omega_{0}\right) \Delta \omega_{2}}
\end{aligned}
$$

using the relation

$$
\dot{F}_{\ell}^{m}(\omega)=i \dot{\phi}_{\ell}^{m}(\omega) F_{\ell}^{m}(\omega)
$$

derived from Eq. (60).

Defining

$$
i \dot{\phi}_{\ell}^{m}\left(\omega_{0}\right) \Delta \omega_{2}=a+b i
$$

we have

$$
\left|\frac{\Delta \omega_{1}}{\Delta \omega_{2}}\right|^{2}=\frac{\mathrm{e}^{2 a}-2 \mathrm{e}^{a} \cos b+1}{a^{2}+b^{2}} .
$$

In the above equation, $\mathrm{e}^{a}$ implies amplitude decay of wave packet, so that $a \leq 0$ is physically constrained. In this region, the ratio is limited as

$$
0<\left|\frac{\Delta \omega_{1}}{\Delta \omega_{2}}\right|^{2} \leq 1
$$

Then, we have the relation

$$
\left|\Delta \omega_{1}\right| \leq\left|\Delta \omega_{2}\right|
$$

This relation implies that the linearization of $\phi_{\ell}^{m}(\omega)$ can be applied in a wider frequency range than in the case of $F_{\ell}^{m}(\omega)$.

\section{References}

Duncan, P. M., A. H. Wang, R. N. Edwards, R. S. Bailey, and G. D Garland, The development and applications of a wide band electromagnetic sounding system using a pseudo-noise source, Geophysics, 45, 1276-1296, 1980.

Hasada, Y., H. Kumagai, and M. Kumazawa, Autoregressive modeling of transfer functions in frequency domain to determine complex travel times, Earth Planets Space, 53, 3-11, 2001.

Kaufman, A. A. and G. V. Keller, Frequency and Transient Soundings, 685 pp., Elsevier, Amsterdam, 1983

Kay, S. M. and S. L. Marple, Spectrum analysis - a modern perspective, Proc. IEEE., 69, 1380-1419, 1981.

Kumazawa, M., Theoretical basis, development and prospect of EM ACROSS - An approach unifying low frequency MT (magnetotellulic) and high frequency radar, Abstracts of international workshop on frontiers in monitoring science and technology for earthquake environments, A2-21, 1998a.

Kumazawa, M., A new light and new eye to look into the solid Earth and a potential monitoring methodology of geodynamics states - introduction to ACROSS, Abstracts of international workshop on frontiers in monitoring science and technology for earthquake environments, A-11, 1998b.

Kumazawa, M., Y. Imanishi, Y. Fukao, M. Furumoto, and A. Yamamoto, A theory of spectral analysis based on the characteristic property of a linear dynamic system, Geophys. J. Int., 101, 613-630, 1990.

Nakajima, T., M. Kumazawa, T. Kunitomo, and K. Tsuruga, Current approach and the preliminary results on the EM ACROSS utilizing the diffusional transmission of electromagnetic signals in the MT range, Abstracts of international workshop on frontiers in monitoring science and technology for earthquake environments, AP2-41, 1998.

Nakajima, T., T. Kunitomo, M. Kumazawa, and Y. Yokoyama, Development of EM-ACROSS and its field test, Bull. Earthq. Res. Inst. Univ. Tokyo, 75, 229-244, 2000 (in Japanese).

Ogawa, K. and M. Kumazawa, Towards the continuous remote sensing of $\mathrm{H}_{2} \mathrm{O}$, tectonic stress and physical states in the Earth's crust by means of acoustic and electromagnetic ACROSS, Proceedings of fall meeting of the Seismological Society of Japan, P45, 1996 (in Japanese).

Oppenheim, A. V. and R. W. Schafer, Digital Signal Processing, 585 pp. Prentice Hall, London, 1975.

Strack, K. M., Exploration with Deep Transient Electromagnetics, 373 pp., Elsevier, Amsterdam, 1992.

Ward, S. H. and G. W. Hohmann, Electromagnetic theory for geophysical applications, in Electromagnetic Methods in Applied Geophysics vol. 1 , 
edited by M. N. Nabighian, pp. 131-312, Society of Exploration Geophysicists, Oklahoma, 1987.

Yokoyama, Y., M. Kumazawa, and T. Nakajima, A decomposition method of electromagnetic transfer function using ray model and autoregressivetype model - the case of horizontal layered structure-, Bull. Earthq. Res. Inst. Univ. Tokyo, 75, 245-263, 2000a (in Japanese).

Yokoyama, Y., M. Kumazawa, T. Kunitomo, and T. Nakajima, Waveform of digital signal for a 3-D electric structure sounding using accurately controlled electromagnetic wave, Bull. Earthq. Res. Inst. Univ. Tokyo, 75, 265-282, 2000b (in Japanese)

Zhdanov, M. S. and G. V. Keller, The Geoelectrical Methods in Geophysical Exploration, 873 pp., Elsevier, Amsterdam, 1994.

Y. Yokoyama, M. Kumazawa, and T. Nakajima (e-mail: takahiro@tono. jnc.go.jp) 